\title{
Paraplegia
}

\section{Estimating the User Population of a Simple Electrical Stimulation System for Standing}

\author{
R. J. Jaeger, PhD, ${ }^{1,2,} 4$ G. M. Yarkony, MD, ${ }^{1,2}$ E. J. Roth, $M D,{ }^{2}$ L. Lovell, \\ BS, ${ }^{3}$ \\ ${ }^{1}$ Pritzker Institute of Medical Engineering, Illinois Institute of Technology, ${ }^{2}$ Rehabili- \\ tation Institute of Chicago, ${ }^{3}$ Midwest Regional Spinal Cord Injury Care System, \\ ${ }^{4}$ Section of Orthopaedics and Rehabilitation Medicine, Pritzker School of Medicine, \\ The University of Chicago, Chicago, Illinois, USA.
}

\begin{abstract}
Summary
Many laboratory demonstrations have been reported on standing or walking with the aid of electrical stimulation. These demonstrations have typically been in small numbers of selected spinal cord injured individuals. The extent to which this technology might ultimately be applicable to the spinal cord injured population at large is not presently known. This study reports estimates of the size of the potential user population of a specific surface electrical stimulation device and protocol. The medical records were reviewed of 192 patients with traumatic thoracic, lumbar, or sacral spinal cord injury resulting in paraplegia. Based on the inclusionary criteria, between 20 and 48 patients $(10.4 \%$ and $25 \%)$ of this sample population could be considered eligible for this surface stimulation protocol. As approximately $45 \%$ of the USA population of spinal cord injured individuals have paraplegia, the results suggest that between $4 \cdot 7 \%$ and $11 \cdot 25 \%$ of all spinal cord injured persons in the USA might be potential users of this particular electrical stimulation technology.
\end{abstract}

Key words: Spinal cord injury; Functional electrical stimulation; Standing.

Functional neuromuscular stimulation (FNS) has been investigated in many laboratories as a potential method of achieving standing (and sometimes walking) in carefully chosen paraplegic individuals (see Cybulski et al., 1984 for a review, also Kralj et al., 1983; Marsolais and Kobetic, 1987; Kralj et al., 1987). Mobility needs are very important in this population (Heinemann et al., 1987). Electrical stimulation has been regarded by some as holding great promise to restore some degree of mobility. Despite the sometimes impressive nature of these demonstrations, there has not yet been complete consensus as to exactly how many spinal cord injured individuals will benefit from this technology.

The present study was designed to estimate the potential number of users of a very specific FNS protocol for standing. The inclusionary criteria for use of this technology in this application are very specific (see Discussion). Transient (5-15 min) periods of standing can be achieved by bilateral electrical stimulation of the quadriceps in individuals with thoracic injuries. Balance aids such as parallel bars 
Table I Number of cases satisfying individual criteria

\begin{tabular}{lcc}
\hline Criterion & $\begin{array}{c}\text { Number } \\
\text { satisfying }\end{array}$ & $\begin{array}{c}\text { Number } \\
\text { not satisfying }\end{array}$ \\
\hline Paraplegic & 192 & 0 \\
T4-T12 lesion & 120 & 72 \\
Absence of UE injury & 164 & 28 \\
KAFO prescribed & 91 & 101 \\
No assoc. med. probs. & 124 & 68 \\
No substance abuse & 135 & 57 \\
Age 18-50 & 162 & 30 \\
\hline
\end{tabular}

or walkers are required. This protocol has been described elsewhere (Cybulski et al., 1984; Yarkony et al., 1987; Jaeger et al,. 1989).

These estimates of the size of the potential user population were obtained by reviewing medical records of 192 spinal cord injured patients to determine the number who met basic user eligibility criteria. The results of this review were then applied to estimates of the size of the national spinal cord injured population in the USA.

\section{Methods}

Based on our previous work, proposed prescription criteria have been established for the successful use of this standing aid by paraplegic patients. These criteria are summarised in Table I and treated more fully in the discussion.

A medical record review was conducted of paraplegic individuals admitted to the Rehabilitation Institute of Chicago (RIC) for the period 1982 through to 1986. A total of 192 records at the time of discharge were reviewed. Data were recorded on a standard form, and entered into a computer database. Items recorded included age (years), sex (male or female), level of injury (lowest intact neurological level), complete or incomplete injury (American Spinal Injury Association, 1982), aetiology (GSW, MVA, fall, diving, or other), status of upper extremities (intact, not intact), knee-ankle-foot orthosis (KAFOs) prescription (prescribed or not prescribed), associated medical problems (presence of hypertension, osteoporosis, and cardiopulmonary disease), and substance abuse (history of alcohol abuse or drug use as determined by the treating physician).

With respect to upper extremity status, patients with fractures or peripheral nerve injury that impaired the use of the upper extremities for assistance in standing up or balancing were considered exclusionary. At the RIC, KAFOs are typically offered to patients who meet skill levels in therapy and have indicated a willingness to use them for standing or ambulation, provided no medical contraindications exist.

\section{Results}

Age, sex, and aetiology

The mean age of this sample of 192 paraplegic individuals was $34 \cdot 4 \pm 15 \cdot 5$ years. A histogram showing the distribution of ages is shown in Figure 1. This 


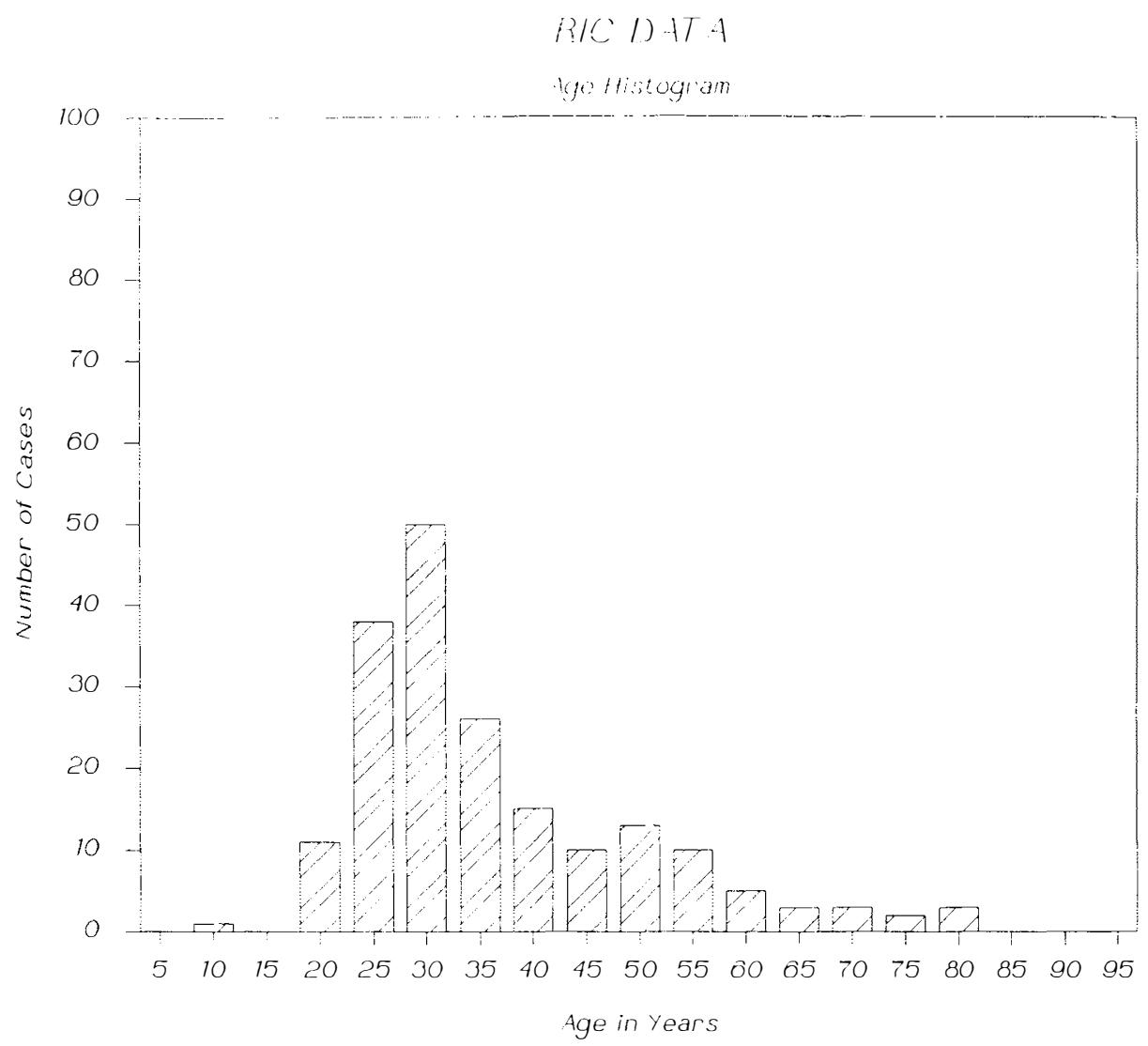

Figure 1 Histogram of age distribution of study sample.

distribution, as well as subsequent sex distribution, aetiologies, and lesion levels may be compared to that for a larger population (Stover and Fine, 1986). The percentage of males and females were $80.5 \%$ and $19.5 \%$, respectively. The aetiologies were gunshot wound $31 \cdot 2 \%$, motor vehicle accident $26 \cdot 6 \%$, fall $21 \cdot 4 \%$, diving $0 \cdot 0 \%$, other $17 \cdot 7 \%$, and unknown $3 \cdot 1 \%$. In this sample $43 \cdot 8 \%$ were complete injuries, $55 \cdot 2 \%$ were incomplete injuries, and $1 \%$ were unknown.

Since the primary criterion for using this protocol was a spinal cord lesion between $\mathrm{T} 4$ and $\mathrm{T} 12$, the distribution of patients with these lesion levels is shown in Figure 2. A total of 120 patients out of the original 192 patients are shown in Figure 2.

\section{Patients meeting individual criteria}

The criteria for the use of this protocol are given in Table I and are followed by the number of cases satisfying the individual criterion. These are individual numbers only and do not reflect interaction between criteria. For example of the 192 patients, 164 had intact upper extremities while 28 had some type of upper 


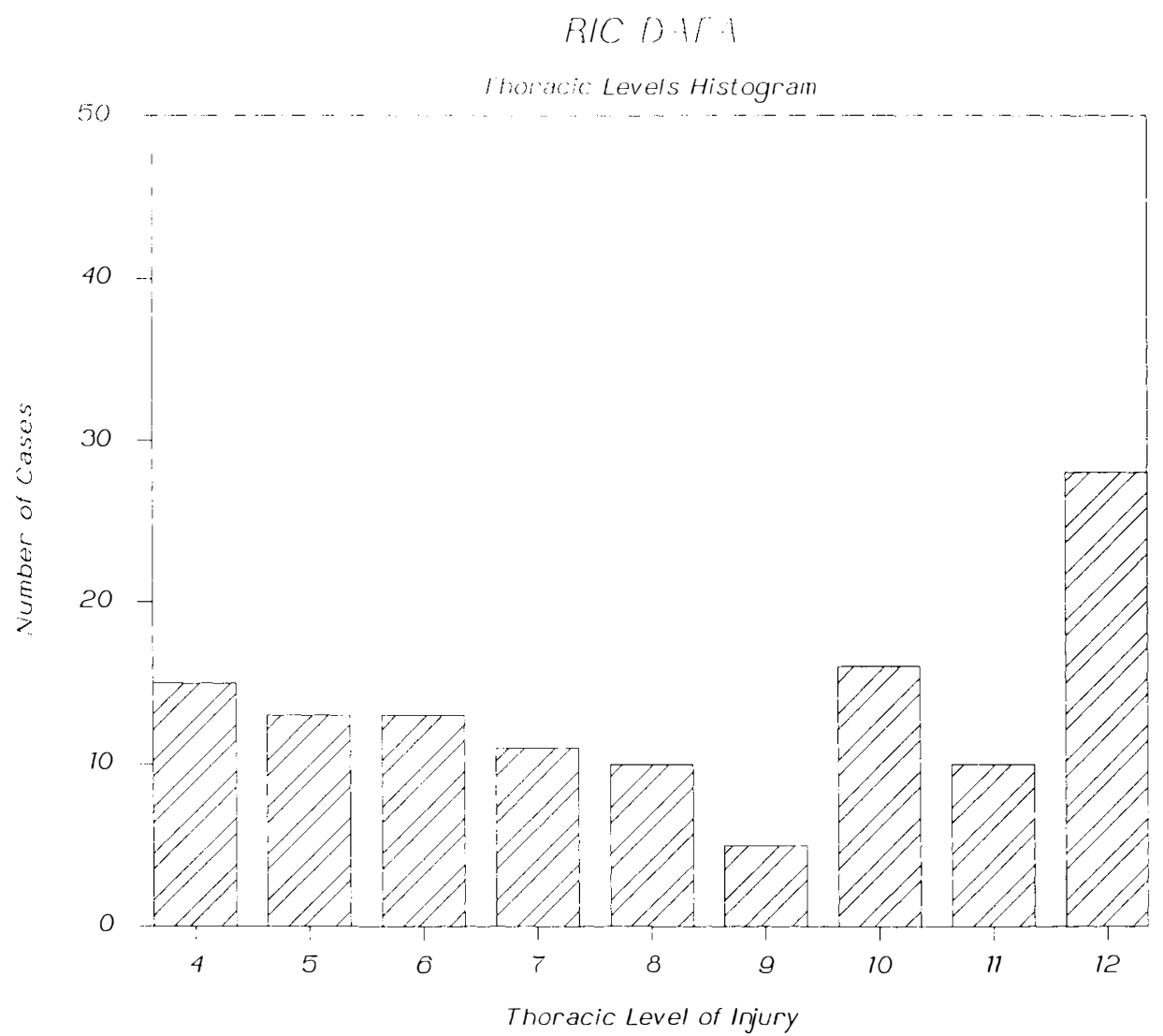

Figure 2 Histogram of lesion level distribution T4-T12.

extremity problem. These 164 patients may or may not satisfy the other criteria. The purpose of Table I is to describe the characteristics of the population with respect to the individual criteria. Table I can be contrasted to Table II, which presents the results of applying the criteria in a sequential manner, as described below.

\section{Use of criteria to establish user population}

The criteria for protocol use were applied to this population in the order indicated in Table II. On each line of the table is the number of subjects remaining after the criterion of that line and all criteria above had been applied to the population, and in parentheses, the number of subjects eliminated by the criterion on the line. The percentage figure is percent of the original 192 subjects remaining. The difference between Tables I and II can be appreciated by considering the criterion of no associated medical problems as an example. In the population of 192 individuals, $124(64 \%)$ had no medical problems (Table I); however in the group of 48 that satisfied the first 4 criteria (Table II) 36 (36 out of 48 or $75 \%$ ) had no medical problems; a figure $10 \%$ better than the entire sample. 
Table II Using criteria to determine user population by elimination of individuals not meeting criteria

\begin{tabular}{lccc}
\hline Criterion & $\begin{array}{c}\text { Number } \\
\text { remaining }\end{array}$ & $\begin{array}{c}\text { Number } \\
\text { eliminated }\end{array}$ & $\begin{array}{c}\text { Percent } \\
\text { remaining }\end{array}$ \\
Paraplegic & 192 & 0 & $100 \%$ \\
T4-T12 lesion & 120 & 72 & $63 \%$ \\
Absence of UE injury & 106 & 14 & $55 \%$ \\
KA FO prescribed & 48 & 58 & $25 \%$ \\
No assoc. med. probs. & 36 & 12 & $19 \%$ \\
No substance abuse & 24 & 12 & $13 \%$ \\
Age 18-50 & 20 & 4 & $10 \%$ \\
\hline
\end{tabular}

From this data the minimum user population of this FNS protocol is $10 \cdot 4 \%$ of paraplegics. If it is assumed that the population using KAFOs was able to stand in spite of medical problems or substance abuse, the estimate may be as high as $25 \%$ of paraplegics. If this minimal $10 \cdot 4 \%$ estimate is applied to the national spinal cord injured population, then between $4 \cdot 7 \%$ and $11 \cdot 25 \%$ of all spinal cord injured individuals would be potential users of this protocol. The national spinal cord injury (SCI) population was estimated to be 45\% paraplegic for 1983-1984 (Stover, and Fine, 1986).

The actual number of patients who could use this protocol depends on the estimate of the total spinal cord injured population size, which is not exactly known (see Cybulski et al., 1984 for review of estimates).

\section{Discussion}

The estimates obtained in this study are functions of the inclusion criteria. The rationale for selection of these criteria is as follows. This protocol requires reasonable trunk balance and stability, and the ability to tolerate the upright posture without autonomic disturbances. Therefore, lesions above T4 are generally not appropriate. Electrical stimulation requires that the stimulated muscle display upper motor neuron paralysis. Since lower motor neuron paralysis of the quadriceps generally is seen below T12, lesions below this level are not appropriate. Balance aids are absolutely essential for this protocol, and some assistance by the upper extremities are needed when standing up and sitting down. Therefore bilateral intact upper extremities are required. Reasonable balance while standing is also a requirement of this protocol. Perhaps the best assessment of this is the patient's ability to use KAFOs. These orthoses are prescribed for both therapeutic standing as well as ambulation. Since the postural stability with the electrical stimulation protocol will be inferior to that obtained with braces, this criterion selects those patients with the necessary standing potential. While there are a number of medical problems that can interfere with the spinal cord injured patient's mobility, hypertension, osteoporosis, and cardiopulmonary disease were deemed contraindications for use of this protocol. Patients who had documented cases of substance abuse (alcohol or drugs) were not chosen to be candidates because of the potential for injury if the electrical stimulation system were used improperly. This $29 \%$ rate of substance abuse in this 196 patient sample is actually 
lower than that reported in other studies (Heinemann et al., 1988). Finally, the age criterion was chosen to exclude older individuals who might be at a higher risk of osteoporosis, and younger individuals for whom the protocol had the potential of being problematic (conflict between young patient and parents desires).

A conservative view of the data presented in this paper suggest that approximately $10 \cdot 4 \%$ (20 out of 192) of all paraplegic individuals in the RIC sample would be potential users of this simple electrical stimulation protocol for standing. This estimate is relatively small. There are a number of factors which might make these numbers even smaller. These include such issues as contractures, primarily at the hip and ankle, spasticity, and psychological problems.

There are also a number of factors which might increase these estimates. For example, it may be possible to treat or control hypertension or other cardiopulmonary problems. Other clinicians may feel that the rather arbitrary age restriction we have imposed could be relaxed, or that relaxing the criteria for KAFOs prescription would be possible. The same is true for substance abuse. Treatment programs for all of the above have varying degrees of efficacy, and it is beyond the scope of this investigation to apply estimates for correction factors to the data presented. If the age, substance abuse, and associated medical problems criteria were not applied, then 48 out of the 192 individuals (25\%) would be potential users.

The desire for new successful mobility enhancing technologies in SCI is great, and functional neuromuscular stimulation is one such technology. It is important, however, that realistic estimates of the user population for such technologies be developed. Despite the uncertainties involved in the estimation process, it appears that a conservative estimate of the user population of the simple standing protocol is in the range of $10 \cdot 4 \%$ to $25 \%$ of all paraplegic individuals, and $4 \cdot 7 \%$ to $11 \cdot 25 \%$ of all spinal cord injured individuals. The present study appears to be the only study of this nature to date in this area, and it should be noted that other protocols for functional neuromuscular stimulation may well have different estimates of potential users.

Restoration of mobility in SCI is an extremely difficult problem. This problem is compounded by individual variations in residual muscle function at particular levels of injury.:Demonstrations of restoring mobility by FNS have been confined to a small number of centers with carefully selected and highly motivated patients. The data from this study could be interpreted to support the view that a number of different protocols for restoring mobility in SCI will be necessary if more than a small portion of the population is to be helped.

\section{Acknowledgements}

This work was supported in part by NIH (SO7RR07027-20, SO7RR07027-21, HL39523, NS20860), NIDRR (G008435046, G008535129). RJJ was supported in part by a Mary E. Switzer Fellowship from NIDRR (133FH70022).

\section{References}

American Spinal Injury Association 1982 Standards for Neurological Classification of Spinal Cord Injury. Chicago, USA. 
Cybulski GR, Penn RD, Jaeger RJ 1984 Lower extremity functional neuromuscular stimulation in cases of spinal cord injury. Neurosurgery 15:132-146.

Heinemann A, Magiera-Planey R, Schiro-Geist C, Gimenes G 1987 Mobility for persons with SCI: and evaluation of two systems. Archives of Physical Medicine and Rehabilitation 50:544-547.

Heinemann A, KeEn M, Donohue R, SChnoll S 1988 Alcohol use by persons with recent spinal cord injury. Archives of Physical Medicine and Rehabilitation 69:619-624.

JAEGER RJ, YARKONY GM, SMITH R 1989 Standing the spinal cord injured patient by electrical stimulation: refinement of a protocol for clinical use. IEEE Transactions on Biomedical Engineering (in press).

KRALJ A, BAJd T, TURK R, BENKo H 1983 Gait restoration in paraplegic patients: a feasibility demonstration using multichannel surface electrode FES. Fournal of Rehabilitation Research and Development 20:3-20.

Kralj A, Bajd T, Turk R, Benko H 1987 Results of FES Application to 71 SCI Patients, Proceedings of RESNA 10th Annual Conference on Rehabilitation Technology (San Jose, CA), pp 645-647.

MARSOLAIS EB, KoBETIC R 1987 Functional electrical sitmulation for walking in paraplegia. Fournal of Bone and foint Surgery 69A:728-733.

Stover SL, Fine PR 1986 Spinal Cord Injury the Facts and Figures. Birmingham Alabama, University of Alabama Birmingham Press.

YARKONY G, JAEGER R, Williamson T et al. 1987 Standing by Functional Neuromuscular Stimulation in the Laboratory and the Home: Case Reports of 18 Paraplegic Individuals RESNA 10th Annual Conference on Rehabilitation Technology June 19-23, 1987, p 608-610. 